\title{
Capacity Scaling of Sparse Mobile Ad Hoc Networks
}

\author{
Michele Garetto \\ Dipartimento di Informatica \\ Università di Torino, Italy
}

\author{
Paolo Giaccone \\ Dipartimento di Elettronica \\ Politecnico di Torino, Italy
}

\author{
Emilio Leonardi \\ Dipartimento di Elettronica \\ Politecnico di Torino, Italy
}

\begin{abstract}
We provide the scaling laws for the transport capacity of a wide class of mobile wireless ad hoc networks. Our analysis generalizes previous results obtained under restrictive assumptions on the node mobility process and overall node density over the network area. The broader family of mobile networks that we consider is able to account for many important characteristics usually recognized in real traces of both human and vehicular mobility. In particular, we consider clustered, sparse networks of heterogeneous nodes, in which the shape of the spatial distribution of each node around one or more home-points plays a fundamental role in determining the overall transport capacity. We identify different operational regimes that arise within our general class of mobile networks, and for each regime we propose optimal scheduling and routing strategies achieving the maximum asymptotic capacity.
\end{abstract}

\section{INTRODUCTION}

Store-and-forward has been for decades the fundamental communication paradigm of packet switched networks, including the first generation of ad hoc wireless networks originally conceived at the end of the last century [1]. The routing protocols developed for these networks have been traditionally based on the assumption that the network topology is always connected, so that, at any time, there exists a path from any sender to its intended receiver. In this context, topological changes due to node mobility have been invariably seen as something evil or at least undesirable, which inevitably degrades the performance achieved in an otherwise stable network topology. In recent years, this traditional view of packet switched networks has changed, as node mobility has become a necessary, fundamental component of a new kind of networks based on a novel store-carry-and-forward communication paradigm (also named "encounter-based forwarding" or "mobility-assisted routing"). According to this new paradigm, relay nodes can physically carry buffered data while they move around the network area, till they get in contact with a suitable next-hop node. Although this scheme incurs much longer delays than the traditional store-and-forward scheme, on the time scale of node movement across the network space, it has laid the foundation of an entire new area of research, usually referred to as Delay Tolerant Networking (DTN) [2], [3], which has recently attracted a lot of attention. Interesting applications include "pocket switched networks" based on human mobility [4], vehicular networks based on public buses [5] or taxicabs [6], sensor networks for wildlife tracking [7], rural kiosks providing Internet access in developing nations [8].

The reason why node mobility plays a fundamental role in DTN is two-fold. Firstly, it permits end-to-end communication in the first place, in the case of sparse, intermittently connected networks suffering from frequent partitioning of the nodes. Indeed, although instantaneous end-to-end routes do not always exist, messages can nevertheless be delivered over time, through the sequence of connectivity graphs generated by nodes' movement. Secondly, mobility can dramatically increase the overall transport capacity of the network, allowing it to scale up to very large number of nodes. Indeed, interference-limited networks of static nodes are known to suffer severe per-node throughput decay (in the order of $1 / \sqrt{n}$ ) as the number of nodes $n$ goes to infinity [9]. In contrast, a simple two-hop relay scheme can keep the per-node throughput constant, under an ideal mobility process in which each node independently and uniformly visits the entire network space [10].

Several routing protocols for sparse delay tolerant networks have been proposed in the literature, which face the challenging problem of selecting good routes in space and time using very limited (and rapidly changing) information about the system state [11]-[15]. However, only few studies have analyzed the fundamental scaling laws of the maximum achievable network capacity in the presence of heterogeneous nodes and under different mobility models. Indeed, the static scenario analyzed by Gupta-Kumar [9] and the 'homogeneous mixing' scenario considered by Grossglauser-Tse [10] are two particular species of a rich zoo of wireless ad hoc networks exhibiting a variety of scaling behavior as we change the mobility patterns of the nodes. The goal of this paper is to provide a comprehensive overview of capacity results for a wide class of mobile networks which accounts for many characteristics largely recognized in real mobility traces of people, animals, and vehicles.

The consideration of the fact that an individual node may not move over the entire network area, but just in a small portion of it, has already pushed some researchers to analyze the impact of restricted mobility models. In [16] the authors extend their results in [10] considering the case where each node independently moves along a randomly chosen great circle on the sphere of unit surface. Quite surprisingly, even under this one-dimensional mobility pattern a constant throughput per source-destination pair can be sustained as the number of nodes increases. In [17] the network of unit area is partitioned into square cells, and nodes are restricted to move within one randomly chosen cell; the authors consider two cases in which the cell area either scales as $(\log n) / n$ or remains constant, obtaining results similar to the scenarios of Gupta-Kumar and Grossglauser-Tse, respectively. The impact of inhomogeneous node density (e.g., node clusters) has been analyzed in [18], [19] for the case of static networks only. In [20], [21] we have addressed the general problem of determining the capacity of mobile networks in the presence of heterogeneous nodes and anisotropic mobility. The contributions of this paper with respect to our previous work will be explained in detail in Section III, after introducing our system assumptions. 


\section{System Assumptions AND NotAtion}

\section{A. Mobility Model}

The family of mobile networks that we consider is denoted by $\mathcal{F}(\alpha, \delta, \nu, \zeta)$ and is characterized by four parameters $\alpha, \delta, \nu, \zeta$. For a given choice of parameters, we build a sequence of networks $\mathcal{F}_{n}$ indexed by the number of nodes $n$, and let $n$ go to infinity. Nodes are supposed to move over a bi-dimensional Torus surface $\mathcal{O}_{n}$ of size $L_{n}$ x $L_{n}$ (to avoid border effects). As we add more and more nodes into the system, the network physical extension $L_{n}$ is expected to increase. To account for this fact, we assume that $L_{n}$ scales with $n$ as $n^{\alpha}$, with $0 \leq \alpha \leq 1 / 2$. The extreme cases are: $\alpha=0$, for which the network area remains constant, and average node density increases linearly with $n ; \alpha=1 / 2$, for which the network area increases linearly with $n$, and average node density remains constant. The first parameter of our network family, $\alpha$, thus determines how the network area (or, alternatively, the average node density) behaves as we increase the number of nodes.

Anisotropic mobility and node heterogeneity are taken into account in our model as follows. First, we observe that, as proven in [20], the transport capacity of a mobile network depends on the mobility pattern only through the stationary distribution of the nodes over the area, under the assumption that the mobility processes of the nodes are jointly stationary and ergodic. In this work, we further assume that nodes move independently of each other. Hence, we only need to characterize the shape of the spatial distribution of individual nodes over region $\mathcal{O}_{n}$. We consider that each node has one or more spots where it is most likely to be found (these can be the private home or the workplace in the case of people, the garage or the warehouse in the case of vehicles, the nest or lair in the case of animals). We refer to such spots with the general term "home-points", and assume that every node has just a finite number of them. At any given time, a node is associated to one of its home-points. The fraction of time during which a node is associated to any of its home-point is supposed to be constant and non null. While being associated to an home-point, a node is not fixed, but moves around it according to a general ergodic process which results into a rotationally-invariant spatial distribution $\phi(d)$ which decays with the distance ${ }^{1} d$ from the home-point as a power law of exponent $\delta$, with $\delta \geq 0$. To avoid problems in proximity of the home-point, we take function $s(d)=\min \left(1, d^{-\delta}\right)$, and normalize it so as to obtain a proper probability density function over the network area: $\phi(d)=\frac{s(d)}{\iint_{\mathcal{O}_{n}} s(d)}$ The precise value of the normalization constant $G=\iint_{\mathcal{O}_{n}} s(d)$ is cumbersome to derive due to the square shape of the network area (as opposed to the circular symmetry of $\phi(d)$ ), however it can be approximated, in order sense ${ }^{2}$, by the following integral in

\footnotetext{
${ }^{1}$ Given any two points $X_{1}=\left(x_{1}, y_{1}\right) \in \mathcal{O}_{n}$ and $X_{2}=$ $\left(x_{2}, y_{2}\right) \in \mathcal{O}_{n}$ we define their distance according to: $d\left(X_{1}, X_{2}\right)=$ $\min _{u, v \in\left\{-L_{n}, 0, L_{n}\right\}} \sqrt{\left(x_{1}+u-x_{2}\right)^{2}+\left(y_{1}+v-y_{2}\right)^{2}}$

${ }^{2}$ Given two functions $f(n) \geq 0$ and $g(n) \geq 0: f(n)=$ $o(g(n))$ means $\lim _{n \rightarrow \infty} f(n) / g(\bar{n})=0 ; f(n)=O(g(n))$ means $\limsup _{n \rightarrow \infty} f(n) / g(n)=c<\infty ; f(n)=\omega(g(n))$ is equivalent to $g(n)=o(f(n)) ; f(n)=\Omega(g(n))$ is equivalent to $g(n)=O(f(n))$; $f(n)=\Theta(g(n))$ means $f(n)=O(g(n))$ and $g(n)=O(f(n))$; at last $f(n) \sim g(n)$ means $\lim _{n \rightarrow \infty} \frac{f(n)}{g(n)}=1$
}

polar coordinates:

$$
G=\Theta\left(\int_{0}^{2 \pi} \mathrm{d} \theta \int_{0}^{L_{n} / 2} \min \left(1, \rho^{-\delta}\right) \rho \mathrm{d} \rho\right)
$$

We obtain that $G$ is finite for any $\delta>2$. For $0 \leq \delta<2$ we have $G=\Theta\left(L_{n}^{2-\delta}\right)$. Let $E[d]$ be the average distance reached by the node from the home-point. The value of $E[d]$ can be approximated (in order sense) by

$$
E[d]=\frac{1}{G} \int_{0}^{2 \pi} \mathrm{d} \theta \int_{0}^{L_{n} / 2} \min \left(1, \rho^{-\delta}\right) \rho^{2} \mathrm{~d} \rho
$$

We have that $E[d]$ is finite for any $\delta>3$. For $2<\delta<3$, it results ${ }^{3} E[d]=\Theta\left(L_{n}^{3-\delta}\right)$, whereas for $0 \leq \delta<2$ we have $E[d]=\Theta\left(L_{n}\right)$.

Parameter $\delta$ accounts for the fact that an individual node does not visit uniformly the network area, but spends most of the time in proximity of a few preferred places, which are assumed to be randomly located within the network region. However, parameter $\delta$ alone does not allow to control the overall node density over the area. In many realistic scenarios it has been found that the node density is largely inhomogeneous, and typically exhibits "concentration points" [6] or hotspots [22] where nodes are more likely to gather. Such clustering behavior has been observed in many different traces related to both human and vehicular movements, and appears to be a quite ubiquitous feature of realistic mobility processes. Concentration points are usually well distinct from each other and fairly stable over time. Examples include dormitories, conference rooms, restaurants, movie theaters, (in the case of people), intersections, parking lots, gas stations (in the case of vehicles), watering holes, oases (in the case of animals).

In our mobility model, we introduce clustering through the distribution of the nodes' home-points. In particular, we consider two different models:

- Uniform: home-points of nodes are uniformly and independently chosen inside area $\mathcal{O}_{n}$.

- Clustered Random: the $n$ nodes are partitioned into $m$ clusters according to i.i.d. random variables. Each cluster has a middle point which is uniformly located within $\mathcal{O}_{n}$. The home-points of nodes belonging to the same cluster are then uniformly and independently placed within a disk of radius $r$ centered at the cluster middle point.

In the Clustered Random model, we let the number of clusters scale with $n$, defining $m=n^{\nu}$, with $0<\nu<1$. The Uniform model can be considered as an extreme case of the Clustered Random model when $\nu=1$ and, in addition, each cluster contains deterministically one home-point. We introduce the cluster density over the area $\rho_{C}=n^{\nu} / n^{2 \alpha}=n^{\nu-2 \alpha}$ and the average inter-distance between cluster middle points $d_{C}=n^{\alpha-\nu / 2}$. Finally, we assume that the cluster radius $r=o\left(d_{C}\right)$, so that, with high probability, clusters do not overlap. Examples of home-point distributions according to Uniform and Clustered Random models are shown in Fig. 1 for $n=10,000$.

In the following we will also consider the Clustered Grid model, which differs from the Clustered Random model in

\footnotetext{
${ }^{3}$ In this paper we will not consider the two special cases $\delta=2$ and $\delta=3$
} 


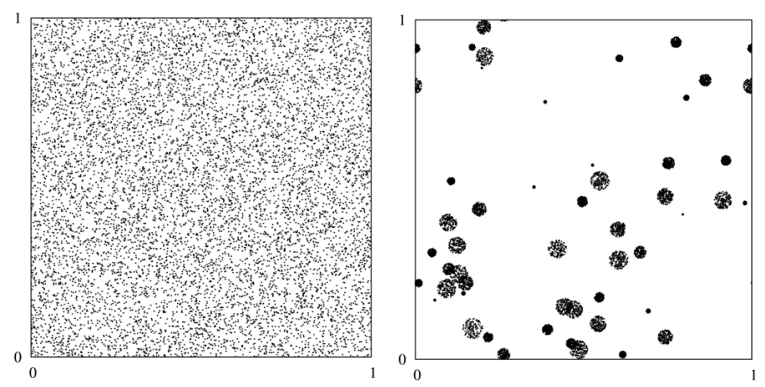

Fig. 1. Examples of home-point distributions according to Uniform mode (left plot) and Clustered Random model (right plot), in the case of $n=10,000$ nodes.

that: i) the cluster middle points are regularly placed over $\mathcal{O}_{n}$, according to a square grid with step $d_{C}$; ii) $r=0$, i.e., all homepoints are co-located at the cluster middle-point. The Clustered Grid model allows to simplify the presentation of some of the ideas of the paper, and constitutes an intermediate step towards the analysis of the Clustered Random model.

At last, we need to describe how we assign multiple homepoints to a given node $i$. To this end, we randomly select one home-point in the area to be the primary home-point of the node, and denote it by $X_{i}^{h}$. Then we specify the probability distribution of the distance $z$ between the primary home-point and any other of its home-points, assuming that the direction is uniformly distributed in $[0,2 \pi]$. Since the number of homepoints of a node is finite, it turns out that all of the gain in terms of network capacity is achieved (in order sense) in the case of just two home-points. Hence, we limit ourselves to considering only one secondary home-point, located at $Y_{i}^{h}$. As an example, the primary home-point could be the residence of a person, and the secondary home-point his/her workplace. A node alternates periods in which it moves around the primary home-point to periods in which it moves around the secondary home-point. Moreover we assume that it travels from one to the other in negligible time and hence does not have the opportunity of exchanging data with other nodes while travelling from one home-point to the other (the contribution of such data exchanges are in any case negligible). If the primary and secondary home-points of a node belong to the same cluster, or, more in general, they are separated by a distance $o\left(d_{C}\right)$, the behavior is the same as in the case of a single homepoint, hence there is no gain in terms of network capacity. The only interesting case is when the distance between the two home-points of a node is $\Omega\left(d_{C}\right)$, therefore we assume that the distribution of the distance $z$ between them is according to $\min \left(1,\left(z / d_{C}\right)^{-\zeta}\right) / Z$, where $Z$ is a proper normalization factor and $\zeta \geq 0$ is one additional parameter of our model. When $\zeta>2$, the average distance $E[z]$ between the two home-points is $\Theta\left(d_{C}\right)$. For $1<\zeta<2, E[z]$ behaves as $\Theta\left(L_{n} / n^{\nu / 2(\zeta-1)}\right)$, whereas for $\zeta<1$ we have $E[z]=\Theta\left(L_{n}\right)$. Therefore, varying $\zeta$ between 1 and 2 , we relate $E[z]$ to the physical network extension, obtaining all intermediate cases between $d_{C}$ (the minimum value for which multiple home-points are beneficial) and $L_{n}$ (equivalent to choosing the secondary homepoint uniformly in the area). Conventionally, $\zeta=-1$ represents the case in which a node has a single home-point, or the average distance between its home-points is $E[z]=o\left(d_{C}\right)$.

Discussion: The class of mobile networks that we study is very general and encompasses a wide range of possible scenarios, while accounting for what we think are ubiquitous features of realistic mobility processes (clustering and power laws in the spatial distribution of nodes). Notice that the Grossglauser-Tse scenario corresponds to the subclass $\mathcal{F}(-, 0,-,-)$ in which $\delta=0$, whereas the other parameters can take any value. The case of static nodes uniformly deployed over the area (GuptaKumar scenario) can be represented by $\mathcal{F}(1 / 2, \infty, 1,-1)$ in which the mobility of each node around a single home-point $(\zeta=-1)$ of the Uniform model $(\nu=1)$ is so limited $(\delta \rightarrow \infty)$ that the node can be considered to be static, while the node density is kept constant $(\alpha=1 / 2)$.

The use of power laws for the node spatial distribution is justified by a number of measurement studies: in [6], the authors analyze a large mobility trace of taxis in the city of Warsaw. The empirical distribution of the number of taxis falling in the cells of a regular grid is found to be heavy-tailed and fairly stable over time. In [4] the duration of contact times between people is found to be heavy-tailed in different traces related to conference and campus-wide experiments. In [23] authors analyze a corporate wireless local area network and find that the fraction of time spent by users with a given access point exhibits a power law.

\section{$B$. Interference Model}

We assume that interference among simultaneous transmissions is described by the protocol model ${ }^{4}$, as introduced in [9], that roughly represents the behavior of CSMA wireless protocols (e.g., 802.11) in the case of omni-directional antennas. According to this model, nodes employ a common range $R_{T}$ for all their transmissions (equivalently, they employ a common power level, i.e., no power adaptation mechanism is used). Node $i$ is allowed to transmit to node $j$ at time $t$, only if: i) the distance between $i$ and $j$ is no more than $R_{T}$, i.e., $d_{i j}(t)<R_{T}$; ii) for every other node $k$ simultaneously transmitting, $d_{k j}(t)>(1+\Delta) R_{T}$, being $\Delta$ a guard factor. We assume that transmissions occur at fixed rate which is normalized to 1 .

\section{Traffic Model}

Similarly to previous work we consider uniform permutation traffic matrices, i.e., traffic patterns in which $n$ randomly selected source-destination pairs $(s, d)$ exchange traffic at rate $\lambda$. Source-destination pairs are selected is such a way that every node is origin and destination of a single traffic flow with average rate $\lambda$. Formally, a uniform permutation traffic matrix is defined by $\boldsymbol{\Lambda}=\lambda\left[\lambda_{s d}\right]$, where $\left[\lambda_{s d}\right] \in\{0,1\}$ and $\sum_{s} \lambda_{s d}=1 \forall s, \sum_{d} \lambda_{s d}=1 \forall d$.

We say that the per-node capacity (or maximum per-node throughput) of the system is $\Theta(h(n))$ if, given a sequence of uniform permutation traffic patterns with rate $\lambda^{(n)}=h(n)$, there exist two constants $c, c^{\prime}$ such that $c<c^{\prime}$ and both the following properties hold:

$$
\left\{\begin{array}{l}
\lim _{n \rightarrow \infty} \operatorname{Pr}\left\{c \lambda^{(n)} \text { is sustainable }\right\}=1 \\
\lim _{n \rightarrow \infty} \operatorname{Pr}\left\{c^{\prime} \lambda^{(n)} \text { is sustainable }\right\}<1
\end{array}\right.
$$

\footnotetext{
${ }^{4}$ Our results can be extended to the physical model of [9]
} 


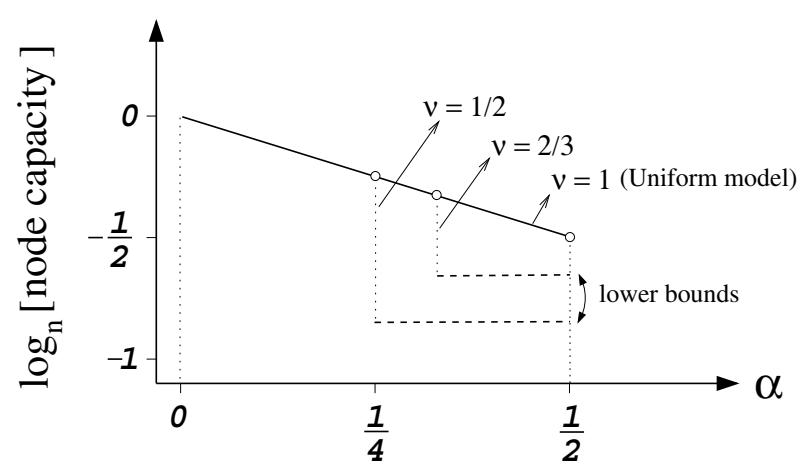

Fig. 2. Capacity results obtained in our previous work

Equivalently, we say in this case that the network capacity (or maximum network throughput) is $\Theta(n h(n))$.

\section{PAPer CONTRIBUtion}

In [21] we have identified two main regimes that arise within our family of mobile networks, under the restriction of a single home-point per node: the super-critical regime, which occurs when $\alpha<\nu / 2$ (i.e., in cohesive networks, where $d_{C} \rightarrow 0$ ), and the sub-critical regime, characterized by $\alpha>\nu / 2$ (i.e., in sparse networks, where $d_{C} \rightarrow \infty$ ). We have then developed a general framework for the analysis of the capacity scaling properties in the super-critical regime. Our main finding, schematically represented in Figure 2, is that the per-node capacity is $\Theta\left(n^{-\alpha}\right)$ with high probability ${ }^{5}$ (w.h.p.), independently of both the shape $\phi(d)$ of the spatial distribution around the home-point (provided that it has finite first moment) and of the parameter $\nu$ of the particular Clustered Random model (including the special case of $\nu=1$, i.e., the Uniform model).

In [21] we have also made a preliminary investigation of the sub-critical regime $(\alpha>\nu / 2)$, limiting our attention to the case in which the node spatial distribution $\phi(d)$ has finite support. In this case, mobility no longer helps, and the per-node capacity is abruptly lowered to a constant value $\Theta\left(n^{\nu / 2-1} / \log n\right.$ ) (for any $\alpha>\nu / 2$ ), equivalent to that of a static network.

The purpose of this paper is to complement and generalize results presented in [20], [21] along several directions: i) offering a complete panorama of system behavior in the subcritical regime for general spatial distributions $\phi(d)$ (Section IV-A); ii) studying the case in which the average distance reached by a node from the home-point is not independent of $n$ (in order sense), but scales itself with the network size, tending to infinity as the number of nodes (or, equivalently, the network physical extension) increases (Section IV-B); iii) extending the analysis to the interesting case in which a node has several home-points variably distributed in the area and moves from one to another over time (Section V).

\section{Single HOME-POINT}

In this section we analyze network scenarios in which $\zeta=-1$, i.e., each node has a single home-point, or, more in general, $E[z]=o\left(d_{C}\right)$. In Section IV-A we analyze the subcritical regime in the case of general spatial distributions for

\footnotetext{
${ }^{5}$ I.e., with a probability tending to 1 as $n \rightarrow \infty$
}

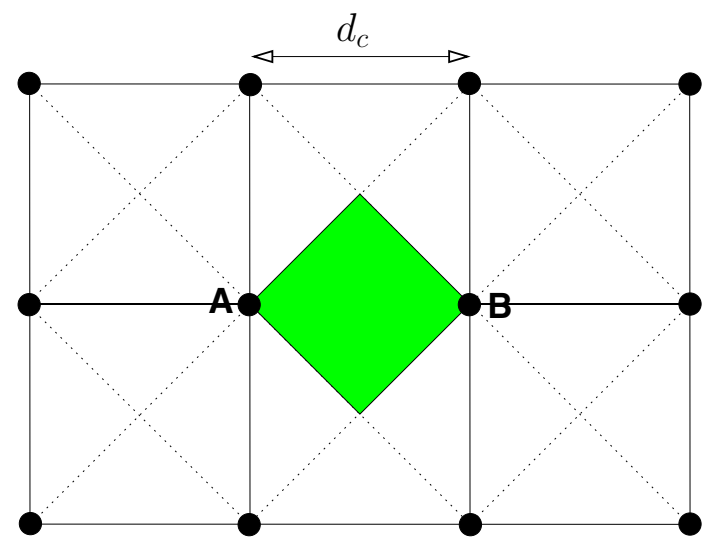

Fig. 3. Regular distribution of clusters over the area and scheduling region between clusters A and B (shaded area)

which the average distance $E[d]$ reached by a node is finite $(\delta>3)$. In Section IV-B we extend the analysis to the case of infinite $E[d](\delta<3)$. A graphical representation of our results is reported and discussed in Section IV-C.

\section{A. Analysis of the sub-critical regime for $\delta>3$}

We remind that the sub-critical regime is characterized by $\alpha>\nu / 2$. In this case, different spatial distributions resulting from the node mobility process can impact the network capacity only in the presence of clustering $(\nu<1)$, otherwise for $\nu=1$ the network behaves as a static one as soon as $\alpha=1 / 2$ (see Figure 2). As a preliminary step, we start considering the Clustered Grid model (IV-A1), which is simpler to analyze. Then we show how our results can be extended to the Clustered Random model (IV-A3).

1) The Clustered Grid model: In this case clusters' centers form a regular square grid, where the distance between neighboring clusters is $d_{C}=n^{\alpha-\nu / 2}$. We now anticipate what is, in this case, the scheduling/routing scheme that achieves the maximum network capacity, and later show in Section IV-A2 that it is not possible to do any better than the proposed scheme.

A source wishing to send a packet to a destination belonging to a different cluster (almost all of the flows), routes it through randomly chosen relay nodes whose home-points belong to an horizontal and/or vertical sequence of adjacent clusters. We call such horizontal/vertical sequence a logical path, because the actual communication between a node and its next hop along the path can take place anywhere in the network, where the two nodes get sufficiently close to each other to enable communication between them. Since the length of each logical hop is equal to $d_{C}$, the average number of hops to reach a destination is $\Theta\left(n^{\nu / 2}\right)$.

Now, let us consider an arbitrary pair of neighboring clusters $A$ and $B$. Transmissions between two nodes whose home-points belong to $A$ and $B$, respectively, are scheduled only in the region of points having either $A$ or $B$ as the closest cluster (shaded area in Figure 3), so as to maximize the density of transmitter-receiver pairs. By so doing, we partition the network into $2 n^{\nu}$ regions of area $\Theta\left(d_{C}^{2}\right)=\Theta\left(n^{2 \alpha-\nu}\right)$, each of them entirely dedicated to scheduling transmissions between nodes whose home-points belong to adjacent clusters. To determine 


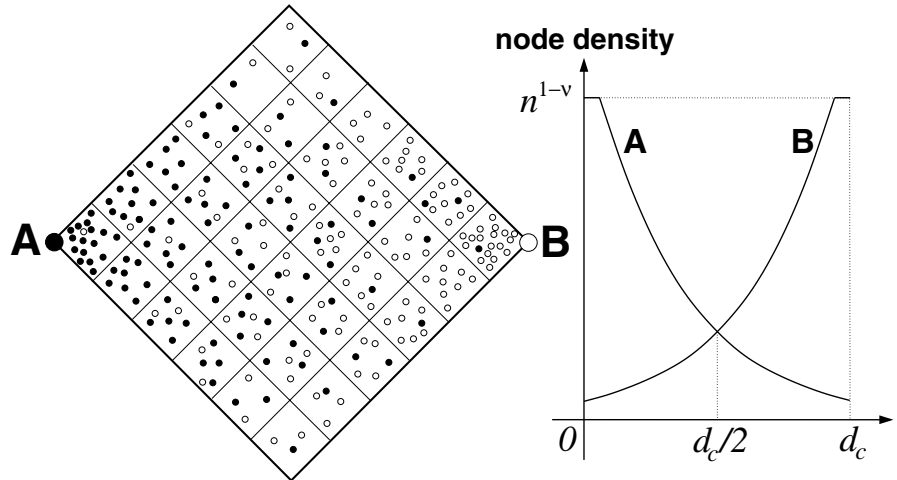

Fig. 4. Density of nodes belonging to clusters A and B in the scheduling region between them

the total number of transmissions that can be scheduled in the network according to the proposed scheme, we make use of the following lemma:

Lemma 1: Let $\sigma>\psi>0$. In a region of area $\Theta\left(n^{\sigma}\right)$, in which the minimum between the density of transmitters and receivers is, at any point, $\Theta\left(n^{-\psi}\right)$, it is possible to schedule $\Theta\left(n^{\sigma-\psi}\right)$ non-interfering transmissions.

Proof: (Lemma 1). We divide the region into a regular tessellation where each element has area $n^{\psi}$. Every element of the tessellation contains one transmitter-receiver pair with probability $\Theta(1)$, thus, by the law of large numbers, there are $\Theta\left(n^{\sigma-\psi}\right)$ elements containing at least one transmitterreceiver pair. To avoid interference, it is sufficient to enable transmissions only in one out of $M=\Theta(1)$ elements of the tessellation, regularly spaced (for example, in the case of a square tessellation, we employ a maximum transmission range $R_{T}=\sqrt{2} n^{\psi / 2}$, and we have $M=9$, assuming a protocol interference model with $\Delta=0$ ).

In our case, the behavior of the density of transmitters and receivers in a given region is illustrated in Figure 4. The density of nodes whose home-points belong to cluster A (or B) behaves as $n^{1-\nu} / d^{\delta}$, where $d$ is the distance from the cluster middlepoint. It is easily seen that the maximum of the minimum density of transmitters and receivers is achieved in the middle of the region, for $d=d_{C} / 2$, which scales in the same way as at $d=d_{C}$, i.e., at the extremes of the region. Hence, at any point, the minimum between the density of transmitters and receivers scales as $\Theta\left(n^{1-\nu} / d_{C}^{\delta}\right)=\Theta\left(n^{1-\nu-(\alpha-\nu / 2) \delta}\right)$. We can thus apply Lemma 1 with $\sigma=2 \alpha-\nu, \psi=1-\nu-(\alpha-\nu / 2) \delta$, obtaining that in each region we can schedule $n^{(\alpha-\nu / 2)(2-\delta)+(1-\nu)}$ non-interfering transmissions. We observe that, in proximity of either $A$ and $B$, there are fewer limitations caused by interference, because nodes coming from the other cluster get surrounded by a large number of nodes belonging to the local cluster, and thus one can use a transmission range small enough to prevent interference with nearby transmissions. However this does not change the scaling order of the number of transmissions that can occur simultaneously, which is any case limited by the minimum between the density of transmitters and receivers.

Notice that in our routing scheme network load is split evenly among the clusters, and since relays are selected at random

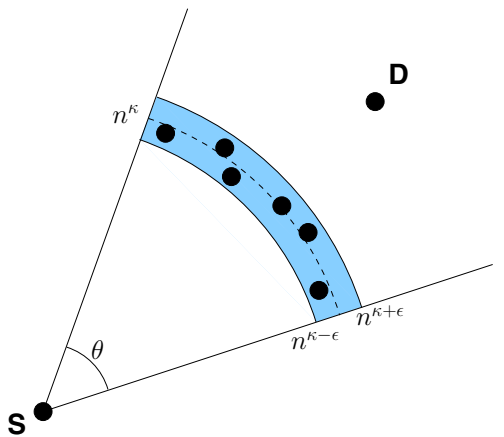

Fig. 5. Next-hop clusters that can be chosen by an alternative routing strategy that selects logical hops of length $\Theta\left(n^{\kappa}\right)$

in each cluster, no node is overloaded. Since there are $\Theta\left(n^{\nu}\right)$ regions, and the average number of hops to reach the destination is $n^{\nu / 2}$, the final per-node capacity, according to the proposed scheduling routing scheme results to be

$$
h(n)=\Theta\left(n^{(\alpha-\nu / 2)(2-\delta)-\nu / 2}\right)
$$

2) A simple proof of optimality: Can we do better than the proposed scheduling/routing scheme for the sub-critical regime? Notice that for any $\delta$ nodes occasionally get in contact with nodes belonging to far away clusters, however in the proposed scheme we only enable communication between nodes whose home-points belong to adjacent clusters (at distance $d_{C}$ ). In this section we provide an intuitive explanation for the fact that no capacity gain can be obtained by allowing transmissions between nodes belonging to clusters which are separated by distance $d=\omega\left(d_{C}\right)$, as long as $\delta>3$. Therefore the proposed scheme is optimal (in order sense). A formal proof of this property is reported in Appendix A.

Let us consider an arbitrary flow between two nodes whose home-points belong to clusters $\mathrm{S}$ and $\mathrm{D}$, respectively (see Figure 5). The typical distance between $S$ and $D$ is of the order of $n^{\alpha}$, the network physical extension. Suppose that we employ logical hops longer than $d_{C}=n^{\alpha-\nu / 2}$. In particular, suppose we enable transmissions between nodes whose homepoints belong to clusters separated by a distance $\Theta\left(n^{\kappa}\right)$, with $\kappa>\alpha-\nu / 2$. To account for all possible logical next-hop at distance $\Theta\left(n^{\kappa}\right)$, we actually consider all clusters located at distances $n^{x}$, with $\kappa-\epsilon<x<\kappa+\epsilon$, where $\epsilon$ is a small constant. In Figure 5 we illustrate the first logical hop according to this alternative routing policy. Notice that we can choose any cluster in a sector of width $\theta<\pi$ (represented by the shaded area in Figure 5) and arrive at the destination in a number of hops equal to $n^{\alpha-\kappa-\epsilon}$ (considering the best case for the number of hops, which is $x=\kappa+\epsilon$ ). The number of clusters in which we can find next-hop nodes is $\Theta\left(\rho_{C} n^{2(\kappa+\epsilon)}\right)=\Theta\left(n^{\nu+2(\kappa+\epsilon-\alpha)}\right)$. We can limit ourselves to the case in which transmissions between nodes belonging to a given cluster and their next-hops occur in a dedicated area of size $\Theta\left(d_{C}^{2}\right)$ around the cluster itself (at any other point between the source and the destination clusters the minimum density of transmitters/receivers is of the same order) and apply again lemma 1 with $\sigma=2 \alpha-\nu, \psi=1+2(\kappa+\epsilon-\alpha)-(\kappa-\epsilon) \delta$, (considering the best case for the density, which is $x=\kappa-\epsilon$ ). 
The resulting upper bound to the per-node throughput according to the alternative scheme, denoted by $h^{\prime}(n)$, is

$$
h^{\prime}(n)=O\left(n^{\kappa(3-\delta)+\epsilon(3+\delta)-\alpha}\right)
$$

which has to be compared with (1). Now, for any $\delta>3$ and any $\kappa>\alpha-\nu / 2$, it is possible to find an $\epsilon$, such that $h^{\prime}(n)=o(h(n))$ : it is sufficient to choose $\epsilon<(\kappa-\alpha+\nu / 2)(\delta-3) /(\delta+3)$. Therefore, exploiting logical hops of any length $d=\omega\left(d_{C}\right)$ provides no gain in terms of network capacity: even in the optimistic situation in which we can schedule such transmissions in addition to those of the previous scheme, the dominant contribution to network capacity is still given by logical hops of length $\Theta\left(d_{C}\right)$.

3) Extension to the Clustered Random model: In the case in which clusters are placed uniformly at random in the area, we partition the network into a regular square tessellation whose elements have area $(16+\delta) n^{2 \alpha-\nu} \log n^{\nu}=\Theta\left(n^{2 \alpha-\nu} \log n\right)$. Standard concentration results based on Chernoff bounds [21] guarantee that, with high probability, the number of clusters falling in each squarelet of the considered tessellation can be replaced by its expected value, which is $\Theta(\log n)$. We adopt again a routing strategy in which data are sent along a logical path formed by a sequence of relay nodes whose home-points belong to adjacent squarelets. A relay node is selected at random in each squarelet. A simple routing scheme according to which data first move horizontally in a row of adjacent squarelets till reaching the column of the destination, and then vertically up to the target node, guarantees that traffic is distributed uniformly in the network and no squarelet is overloaded. The average number of hops of the proposed routing scheme is $\Theta\left(n^{\nu / 2} / \sqrt{\log n}\right)$. A lower bound on the number of transmissions that can be scheduled in each squarelet can be obtained if one enables transmissions between a node and its next hop along the route at any point of the two adjacent squarelets, irrespective of the actual locations of the home-points of the nodes within the squarelets. Using this simple scheduling policy, the number of transmissions that can be enabled in each squarelet can be computed using the same rationale of Lemma 1. Since the typical distance between clusters belonging to adjacent squarelets is $d_{H}=\Theta\left(n^{\alpha-\nu / 2} \sqrt{\log n}\right)$, the minimum density of transmitters/receivers at any point is of the order of $n^{1-\nu} / d_{H}^{\delta}$, and thus in each squarelet one can schedule $\Theta\left(n^{(\alpha-\nu / 2)(2-\delta)+1-\nu}(\log n)^{1-\delta / 2}\right)$ transmissions. The final per-node capacity turns out to be

$$
h(n)=\Theta\left(n^{(\alpha-\nu / 2)(2-\delta)-\nu / 2}(\log n)^{\frac{1-\delta}{2}}\right)
$$

which differs from the one computed in the case of a regular grid of clusters (1) for the factor $(\log n)^{\frac{1-\delta}{2}}$.

\section{B. The case of $\delta<3$}

When $\delta<3$, the average distance reached by a node from its home-point is no longer finite, but scales with $n$, as described in Section II-A. Moreover, in this case Equation (2) suggests that long logical hops are better off than short logical hops. Actually, according to (2), to maximize the capacity one should exploit maximum-length logical hops to reach the destination (i.e., maximize $\kappa$ ), thus delivering data over routes comprising only a finite number of hops, i.e., the average number of hops is $\Theta(1)$.

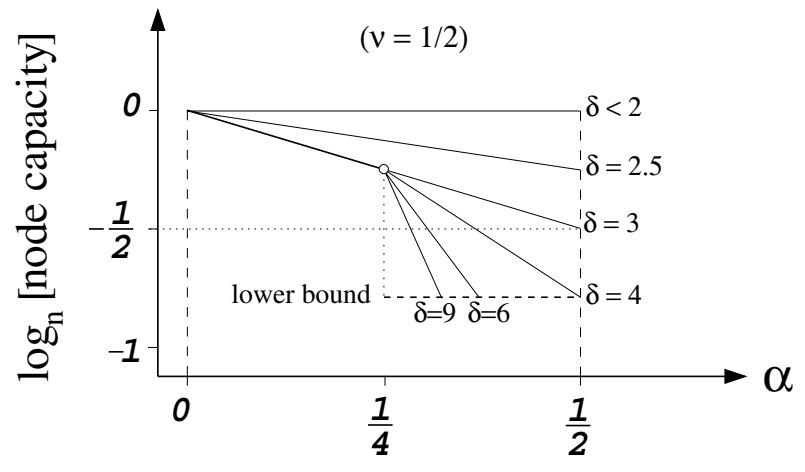

Fig. 6. Illustration of capacity results in the case of single home-point, for the particular value $\nu=1 / 2$

The simplest scheduling/routing scheme, in this regime, is the well known 2-hops relay scheme proposed by GrossglauserTse. Notice that, when $\delta$ goes below the critical value of 3 , the optimal routing scheme shifts abruptly from using the shortest hops to using the longest hops, without intermediate regimes.

We now evaluate how the network capacity scales in the case of $\delta<3$. Essentially, we need to determine how many transmissions can be scheduled between nodes whose homepoints are separated by a distance $n^{\alpha}$ (almost all flows require at least one logical hop of length $\Theta\left(L_{n}\right)$, the physical network extension). Using the same arguments as before, we can limit ourselves to a scheduling policy in which transmissions of nodes belonging to a given cluster are enabled only in the region of area $\Theta\left(n^{2 \alpha-\nu}\right)$ around the cluster itself. Notice that there are $\Theta\left(n^{\nu}\right)$ clusters that can be exploited as next-hop to reach a destination at the typical distance $n^{\alpha}$.

We can thus apply again lemma 1 with $\sigma=2 \alpha-\nu, \psi=$ $1-\alpha \delta-\log G$, where $G$ is the normalization constant of the spatial distribution of a node, which can scale with $n$ as well (Section II-A). The resulting per-node capacity is given by

$$
h(n)=\Theta\left(\frac{n^{\alpha(2-\delta)}}{G}\right)
$$

Now, for $0<\delta<2$, we have $G=\Theta\left(L_{n}^{2-\delta}\right)$, and we obtain a per-node capacity $\Theta(1)$, the same as in the Grossglauser-Tse case. For $2<\delta<3, G$ is finite, thus the per-node capacity scales as $\Theta\left(n^{\alpha(2-\delta)}\right)$.

\section{Graphical representation of capacity results}

A schematic representation of the capacity results derived in previous sections is illustrated in Figure 6, for the case $\nu=1 / 2$. For $\delta<3$, the capacity is independent of $\nu$, and therefore there are no super- or sub-critical regimes, but a single operational regime for all values of $(\alpha, \nu)$. When $\delta=3$ the capacity is the same as in the super-critical regime, i.e., $n^{-\alpha}$. When $\delta=4$, at $\alpha=1 / 2$ we get (for any $\nu$ ) the same capacity as the lower bound of a static network, i.e., $n^{\nu / 2-1} / \log n$. In general, if $\delta<4$ (and $\alpha<1 / 2$ ) the scheme proposed in Section IV-A for the sub-critical regime allows to achieve a better capacity than the one obtained in a static network. If $\delta>4$, there is a value $\alpha^{*}$ after which mobility can not be anymore exploited to increase the network throughput. In such cases network capacity is achieved by a scheme that ignores mobility (i.e., 
according to which $R_{T}=\Theta\left(d_{C}\right)$ ). The critical value of $\alpha$ turns out to be $\alpha^{*}=\frac{2-4 \nu+\nu \delta}{2(\delta-2)}$.

\section{TWO HOME-POINTS}

We now extend our analysis to the case of $\zeta \geq 0$, in which every node has two home-points located at distinct clusters (i.e., at distance $\left.\Omega\left(d_{C}\right)\right)$. As we will see, in this case it is possible to achieve a capacity gain provided that the average distance between the primary and the secondary home-point of a node is larger (in order sense) than the average distance reached by a node around one home-point, i.e., $E[z]=\omega(E[d])$.

\section{A. The Clustered Grid Model}

Similarly to what we have done before, we first describe the scheduling/routing scheme that achieves the maximum network capacity, and later show how to prove its optimality. We consider a forwarding strategy according to which only intra-cluster transmissions are allowed, i.e., transmission among nodes whose home-points belong to the same cluster. Notice that information can be effectively transferred from one cluster to another when nodes migrate from their primary to their secondary home-points. Indeed, the periodic migrations between the two home-points of a node permit to establish a "virtual tunnel" of capacity $\Theta(1)$, through which information can be conveyed. Moreover a communication link of capacity $\Theta\left(n^{-\nu}\right)$ exists between every pair of nodes belonging to the same cluster [20], [21]. We observe that this strategy is somehow the opposite of the one proposed in Section IV for the single homepoint case, where only transmissions between nodes belonging to different clusters are scheduled.

The system can be represented by a graph $G(V, E)$ with $|V|=2 n$, in which a couple of vertices $v_{i}^{1}, v_{i}^{2}$ is associated to each node $i$ of the network. Graph $G(V, E)$ is embedded into the three dimensional domain $\mathcal{O}_{n} \times[1, n]$, being vertices $v_{i}^{1}$ and $v_{i}^{2}$ respectively placed at points $\left(X_{i}^{h}, i\right)$ and $\left(Y_{i}^{h}, i\right)$. An edge of unitary capacity joins $v_{i}^{1}$ and $v_{i}^{2}$. Edges of capacity $n^{-\nu}$ join pair of vertices belonging to the same cluster (i.e. with the same projection on $\mathcal{O}_{n}$ ).

We now compute an upper bound on the network transport capacity adopting a standard technique that resorts to the evaluation of the capacity of graph cut-sets (see Appendix B). In our case we consider the cut-set induced by a vertical plane (i.e., orthogonal to $O_{n}$ ) that divides the network area $\mathcal{O}_{n}$ in two congruent parts $\mathcal{A}$ and $\mathcal{B}$. We observe that the capacity crossing the considered plane, denoted by $\mu_{\text {cross }}$, by construction equals the number of nodes having one home-point in $\mathcal{A}$ and one in $\mathcal{B}$; thus, $\mu_{\text {cross }}$ is a random variable over the $\sigma$-field defined by the location of the nodes' home-points; however, using standard concentration results (see [21]), it can be shown that, w.h.p., when $n \rightarrow \infty$ this random variable takes a value which is equal, in order sense, to its mean (averaged over all configurations of home-points in the area).

The computation of $E\left[\mu_{\text {cross }}\right]$ can then be reduced to the evaluation of the probability that a node having its primary home-point in $\mathcal{A}$ has its secondary home-point in $\mathcal{B}$. We have:

$$
\begin{array}{r}
E\left[\mu_{\text {cross }}\right]=2 n \operatorname{Pr}\left\{Y_{i}^{h} \in \mathcal{B}, X_{i}^{h} \in \mathcal{A}\right\}= \\
4 \int_{\mathcal{A}} \operatorname{Pr}\left\{Y_{i}^{h} \in \mathcal{B} \mid X_{i}^{h}=(x, y) \in \mathcal{A}\right\} \rho_{0} \mathrm{~d} x \mathrm{~d} y
\end{array}
$$

where $\rho_{0}=n^{1-2 \alpha}$ is the average node density within $\mathcal{O}_{n}$. After some calculations it results:

$$
\mu_{\text {cross }}= \begin{cases}\Theta(n) & \zeta<1 \\ \Theta\left(n^{1+(1-\zeta) \nu / 2}\right) & 1<\zeta<2 \\ \Theta\left(n^{1-\nu / 2}\right) & \zeta>2\end{cases}
$$

Dividing by the number of source/destination traffic relations whose source home-point lies in $\mathcal{A}$ and destination home-point in $\mathcal{B}$, which is $\Theta(n)$, we obtain the following upper bounds on the per-node capacity:

$$
\hat{h}(n)= \begin{cases}\Theta(1) & \zeta<1 \\ \Theta\left(n^{(1-\zeta) \nu / 2}\right) & 1<\zeta<2 \\ \Theta\left(n^{-\nu / 2}\right) & \zeta>2\end{cases}
$$

It can be shown that no tighter bound can be found by selecting other surfaces (for example horizontal planes) and computing the capacity crossing the surface. Thus, exploiting the result in Appendix B, we obtain that a routing/forwarding scheme exists such that the per-node capacity is

$$
h(n)=\Omega\left(\frac{\hat{h}}{\log n}\right)
$$

Comparing the result in (5) with that in (4), we see that, when $E[d]$ scales with $n(\delta<3)$, the case of two home-points provides a higher capacity, in order sense, than in the case of a single home-point provided that $(1-\zeta) \nu / 2>\alpha(2-\delta)$. This is precisely the condition under which the average separation between primary and secondary home-points is larger than the average distance reached by a node around one home point (see expressions of $E[z]$ and $E[d]$ in Section II-A). For the same reason, since $E[d]=\Omega(1)$, we observe that the case of twohome-points is beneficial only when $E[z]$ does not tend to zero as $n$ increases. In particular, for $\zeta \geq 2$, this implies that we must be in the case of $\alpha>\nu / 2$, i.e., in the sub-critical regime.

We remark that it can be rather easily verified that allowing transmissions between nodes belonging to different clusters does not increase the overall capacity: even if inter-cluster transmissions could be scheduled in addition to intra-cluster ones, the dominant contribution would still be given by a scheme employing only intra-cluster transmissions.

\section{B. Extension to Cluster Random and Uniform models}

The above results can be extended to the case of the Cluster Random model. The extension is trivial when $r=\Theta(1)$, i.e., the physical span of clusters does not scale with $n$. Indeed, in this case, even if nodes belonging to the same cluster do not share anymore the same home-point, they frequently come in contact for effect of mobility. A virtual communication link of capacity $\Theta\left(n^{-\nu}\right)$ is still established between every pair of nodes belonging to the same cluster, by the adoption of simple scheduling policies. Thus the scheme described in the previous section allows to achieve the same capacity results.

When $r=\omega(1)$, contacts among nodes belonging to the same cluster become more sporadic, thus potentially determining a degradation of system performance; this because capacities among nodes belonging to the same cluster are not anymore homogeneously equal (in order sense) to $\Theta\left(n^{-\nu}\right)$. ¿From an analysis of the system, however, it emerges that, for any $\nu<1$, the intra-cluster transport capacity never becomes 
the network bottleneck (i.e., cut-sets induced by horizontal planes of $G(V, E)$ do not provide tighter limits to the network capacity w.r.t. to vertical planes).

For the Uniform model $(\nu=1)$, instead, a small throughput degradation of order $\log n$ occurs in the case $\zeta<1$ and $\alpha=1 / 2$. We have:

$$
\hat{h}(n)= \begin{cases}\Theta(1) & \zeta<1, \alpha<1 / 2 \\ \Theta(1 / \log n) & \zeta<1, \alpha=1 / 2 \\ \Theta\left(n^{(1-\zeta) / 2}\right) & 1<\zeta<2 \\ \Theta\left(n^{-1 / 2}\right) & \zeta>2\end{cases}
$$

In the Uniform model, a scheme that obtains optimal throughput can be devised by dividing the domain $\mathcal{O}_{n}$ into squarelets of size $1 \times 1$ (for $\alpha<1 / 2$ ) or $4 \sqrt{\log n} \times 4 \sqrt{\log n}$ (for $\alpha=1 / 2$ ), and allowing transmissions only between nodes whose home-points reside in the same squarelet (in practice, nodes whose home-points fall within the same squarelet can be thought to belong to a virtual cluster equivalent to those of the Random Cluster Model). When $\alpha<1 / 2, \Theta\left(n^{1-2 \alpha}\right)$ nodes fall within each squarelet; in addition, being the squarelets of unitary surface, nodes whose home-points belong to the same squarelet come frequently in contact; as a consequence a virtual communication link of capacity $\Theta\left(1 / n^{1-2 \alpha}\right)$ exists between every pair of nodes within the same squarelet (to apply Lemma 1 , a transmission range $R_{T}=n^{\alpha-1 / 2}$ is to be selected in this case). When $\alpha=1 / 2$, the number of nodes within each squarelet is $\Theta(\log n)$; in this case, a transmission range $R_{T}=\sqrt{\log n}$ must be selected. This because there are nodes in the network whose home-points have minimum distance $\Omega(\log n)$ from any other node home-point; as a result, the capacity of the virtual communication link between node pairs within the same squarelet becomes $\Theta\left(1 / \log ^{2} n\right)$ for effect of interference among transmissions.

\section{CONCLUSIONS}

In this paper, we have considered clustered, sparse networks of heterogeneous mobile nodes, in which the shape of the spatial distribution of each node around one or more homepoints plays a fundamental role in determining the overall transport capacity. Complementing results in [21], we have offered a complete view of the system behavior in the subcritical regime, for general spatial distributions. We have also analyzed the case in which the average distance reached by a node from a home-point is not independent of $n$ (in order sense), but scales itself with the network size. At last, we have extended the analysis to the interesting case in which a node has two or more home-points variably distributed over the network area and moves from one to another over time.

\section{REFERENCES}

[1] J. Broch, D. A. Maltz, D. B. Johnson, Y.-C. Hu and J. Jetcheva, "A Performance Comparison of Multi-Hop Wireless Ad Hoc Network Routing Protocols", in Proc. MobiCom '98, Dallas, TX, October 1998.

[2] Delay Tolerant Network Research Group (DTNRG). www.dtnrg.org

[3] K. Fall, "A Delay-Tolerant Network Architecture for Challenged Internets", In Proc. ACM SIGCOMM, 2003.

[4] A. Chaintreau, P. Hui, J. Crowcroft, C. Diot, R. Gass, J. Scott, "Impact of Human Mobility on the Design of Opportunistic Forwarding Algorithms", in Proc. IEEE INFOCOM '06, Barcelona, Spain, April 2006.
[5] J. Burgess, B. Gallagher, D. Jensen, B. N. Levine, "MaxProp: Routing for Vehicle-Based Disruption-Tolerant Networking", in Proc. IEEE INFOCOM 2006, Barcelona, Spain, April 2006.

[6] N. Sarafijanovic-Djukic, M. Piorkowski, and M. Grossglauser, "Island Hopping: Efficient Mobility-Assisted Forwarding in Partitioned Networks", IEEE SECON 2006, Reston, VA, Sep. 2006.

[7] P. Juang, H. Oki, Y. Wang, M. Martonosi, L.-S. Peh, D. Rubenstein, "Energy-Efficient Computing for Wildlife Tracking: Design Tradeoffs and Early Experiences with ZebraNet", in Proc. ASPLOS-X, October, 2002.

[8] A. Seth, D. Kroeker, M. Zaharia, S. Guo, S. Keshav, "Low-cost Communication for Rural Internet Kiosks Using Mechanical Backhauls," in Proc. ACM MobiCom '06, Los Angeles, CA, September 2006.

[9] P. Gupta, P.R. Kumar, "The capacity of wireless networks", IEEE Trans. on Information Theory, vol. 46, n.2, pp. 388-404, Mar. 2000.

[10] M. Grossglauser, D.N.C. Tse, "Mobility increases the capacity of ad hoc wireless networks", IEEE Trans. on Networking, vol. 10, n.2, pp. 477486, Aug. 2002.

[11] S. Jain, K. Fall, and R. Patra, "Routing in a delay tolerant network", in Proc. ACM SIGCOMM, 2004.

[12] R. C. Shah, S. Roy, S. Jain and W. Brunette, "Data MULEs: Modeling and analysis of a three-tier architecture for sparse sensor networks", Elsevier Ad Hoc Networks Journal, Vol. 1, No. 2-3, pp. 215-233, Sept. 2003.

[13] W.Zhao, M.Ammar, and E.Zegura, "A message ferrying approach for data delivery in sparse mobile ad hoc networks", in Proc. MobiHoc '04

[14] M. Grossglauser and M. Vetterli, "Locating Mobile Nodes with EASE: Learning Efficient Routes from Encounter Histories Alone", IEEE/ACM Trans. on Networking, vol. 14, no. 3, June 2006.

[15] A. Lindgren, A. Doria, and O.Schelen, "Probabilistic routing in intermittently connected networks", Mobile Computing and Communications Review, vol.7, n.3, pp.19-20, 2003.

[16] S.N. Diggavi, M. Grossglauser, D.N.C. Tse, "Even one-dimensional mobility increases ad hoc wireless capacity", IEEE Trans. on Information Theory, vol. 51, n. 11, pp. 3947-3954, Nov. 2005.

[17] R. M. Moraes, H. R. Sadjadpour and J. J. Garcia-Luna Aceves, "MobilityCapacity-Delay Trade-off in Wireless Ad Hoc Networks," Elsevier Journal on ad hoc networks, July 2005.

[18] S. R. Kulkarni, P. Viswanath, "A Deterministic Approach to Throughput Scaling in Wireless Networks," IEEE Trans. on Information Theory, vol. 50, no. 6 , pp. 1041-1049, June 2004

[19] S. Toumpis, "Capacity bounds for three classes of wireless networks: asymmetric, cluster, and hybrid", in Proc. ACM MobiHoc '04, pp. 133144, Tokyo, Japan, May 2004.

[20] M. Garetto, P. Giaccone, E. Leonardi, "On the Capacity of Ad Hoc Wireless Networks Under General Node Mobility,' in Proc. IEEE Infocom '07, Anchorage, AK, May 2007.

[21] M. Garetto, P. Giaccone, E. Leonardi, "Capacity Scaling in Delay Tolerant Networks with Heterogeneous Mobile Nodes" in Proc. ACM MobiHoc '07, Montréal, QC, Canada, September 2007.

[22] M. Kim, D. Kotz, S. Kim, "Extracting a mobility model from real user traces", in Proc. IEEE INFOCOM '06, Barcelona, Spain, April 2006.

[23] M. Balazinska, P. Castro, "Characterizing Mobility and Network Usage in a Corporate Wireless Local-Area Network", in Proc. MobiSys 2003, San Francisco, CA, May 2003.

[24] Y. Aumann and Y. Rabani, "An $O(\log k)$ Approximate Min-Cut MaxFlow Theorem and Approximation Algorithm", SIAM Journal on Computing, vol. 27, n. 1, 1998.

\section{APPENDIX A \\ PROOF OF OPTIMALITY}

An abstract representation of the network is provided by a capacitated di-graph $G(V, E, \mu)$, (i.e. a directed graph in which a non-null capacity $\mu_{i j}$ is associated to every edge $(i, j)$ ). Vertex $i \in V$ corresponding to node $i$ is placed at home-point $X_{i}^{h}$. Quantities $\mu_{i j}$ represent the long term mean rate at which data are sent from node $i$ to node $j$ according to the adopted scheduling/routing strategy.

Information flow $s \rightarrow d$ can follow different paths in $G(V, E, \mu)$ from $s$ to $d$, each path corresponding to a different sequence of intermediate relay nodes. 
Proposition 1: Defined $d_{i j}^{h}=\left\|X_{i}^{h}-X_{j}^{h}\right\|$, if traffic $\Lambda$ is sustainable, then:

$$
\lambda \sum_{s d} \lambda_{s d} d_{s d}^{h} \leq \sum_{i j} \mu_{i j} d_{i j}^{h}
$$

Proof: The proof descends from the continuity constraints on routing. Indeed for any source-destination pair $(s, d)$, traffic $\lambda_{s d}$ must be routed in graph $G(V, E, \mu)$ through paths connecting $s$ and $d$. The length of these paths, by triangular inequality, cannot be shorter than $d_{s d}^{h}$, thus the assert follows.

We introduce the cumulative quantity

$$
T(d)=-\sum_{i} \sum_{j} \mu_{i j} \mathbb{I}_{d_{i j}^{h}>d}
$$

representing the average aggregate rate exchanged among all nodes $i$ and $j$, such that $d_{i j}^{h}>d$. From its definition $T(d+$ $\Delta)-T(d)=\sum_{i} \sum_{j} \mu_{i j} \mathbb{I}_{d<d_{i j}^{h}<d+\Delta}$, and thus

$$
\sum_{i} \sum_{j} \mu_{i j} d_{i j}^{h}=\int x \mathrm{~d} T(x)
$$

As a consequence, if we are able to lower bound $T(d)$ with a function $S(d)$, with $S\left(L_{n}\right)=0$ (i.e., to find $S(d)$ such that $T(d) \geq S(d) \forall d)$, then we are able to upper bound the network throughput. Indeed, by integration per parts it can be easily shown that:

$$
\int x \mathrm{~d} T(x) \leq \int x \mathrm{~d} S(x)
$$

which implies:

$$
\lambda \sum_{s d} \lambda_{s d} d_{s d}^{h} \leq \int x \mathrm{~d} S(x)
$$

In our case, for sufficiently large $n$, being $d_{s d}^{h}=\Theta\left(n^{\alpha}\right)$, w.h.p. it results:

$$
\lambda=O\left(\frac{1}{n^{\alpha+1}} \int x \mathrm{~d} S(x)\right)
$$

To obtain an expression for $S(d)$, consider two mobiles $i$ and $j$ whose home-points are $X_{i}^{h}$ and $X_{j}^{h}$, and let $\left\|X_{i}^{h}-X_{j}^{h}\right\|=d_{i j}$; the event $\left\{d_{i j}(t)<R_{T}\right\}$, by construction

$$
\begin{aligned}
\left\{d_{i j}(t)<R_{T}\right\} \subseteq\{ & \left.\left\|X_{i}(t)-X_{i}^{h}\right\|>\frac{d-R_{T}}{2}\right\} \cup \\
& \left\{\left\|X_{j}(t)-X_{j}^{h}\right\|>\frac{d-R_{T}}{2}\right\}
\end{aligned}
$$

i.e.,

$$
\begin{array}{r}
\operatorname{Pr}\left\{d_{i j}(t)<R_{T}\right\} \leq \operatorname{Pr}\left\{\left\|X_{i}(t)-X_{i}^{h}\right\|>\frac{d-R_{T}}{2}\right\}+ \\
\operatorname{Pr}\left\{\left\|X_{j}(t)-X_{j}^{h}\right\|>\frac{d-R_{T}}{2}\right\}= \\
2 \operatorname{Pr}\left\{\left\|X_{i}(t)-X_{i}^{h}\right\|>\frac{d-R_{T}}{2}\right\}=2 \int_{\mathcal{O}_{n}} \phi(x) \mathbb{I}_{x>\frac{d-R_{T}}{2}} \mathrm{~d} x
\end{array}
$$

Indeed, the aggregate number of transmissions $T(d)$ that can potentially occur between nodes whose home-points are separated by a distance greater than or equal to $d$, satisfies:

$$
T(d) \geq-\sum_{i} E\left[\mathbb{I}_{\left\|X_{i}(t)-X_{i}^{h}\right\|>\frac{d-R_{T}}{2}}\right]
$$

since for any such transmission at least one of the two nodes must be at a distance greater than or equal to $\frac{d-R_{T}}{2}$ from its home-point. Therefore,

$$
T(d) \geq-2 n \int_{\mathcal{O}_{n}} \phi(x) \mathbb{I}_{x>\frac{d-R_{T}}{2}} \mathrm{~d} x=S(d)
$$

Now, considering that the minimum distance between nodes belonging to different clusters is $d=d_{C}=n^{\alpha-\nu / 2}$, and that $R_{T}=o(d)$, we obtain as upper-bound to the per-node capacity ${ }^{6}$

$$
\lambda=O\left(n^{(\alpha-\nu / 2)(2-\delta)-\nu / 2}\right)
$$

which proves the optimality (in order sense) of the scheduling/routing scheme described in section IV-A for $\delta>3$.

The same reasoning can be employed to prove the optimality of the scheduling/routing scheme for the case of $\delta<3$, and for the Clustered Random model.

\section{APPENDIX B}

\section{GRAPHS CUT-SETS AND NETWORK CAPACITY}

Consider a communication network admitting a representation in terms of an associated capacitated di-graph $G(V, E, \mu)$, i.e., a directed graph in which a non-null capacity $\mu_{i j}$ is associated to every edge $(i, j)$.

The maximum transport capacity of the network under traffic matrix $\boldsymbol{\Lambda}=\lambda\left[\lambda_{s d}\right]$, can be formalized in terms of a Maximum Concurrent Flow (MCF) problem [24] over $G(V, E, \mu)$, i.e., in terms of the following multi-commodity flow problem over $G(V, E, \mu)$.

Having defined for every flow $(s, d)$, and every $(i, j) \in E$ variables $f_{i j}^{s d} \in[0,1]$, representing the average fraction of traffic from node $s$ to node $d$, which is routed through edge $(i, j)$, find:

$$
\max \lambda
$$

subject to the constraints:

$$
\begin{gathered}
\lambda \sum_{s} \sum_{d} \lambda_{s d} f_{i j}^{s d} \leq \mu_{i j} \\
\sum_{i} f_{i j}^{s d}-\sum_{k} f_{j k}^{s d}=\left\{\begin{array}{cc}
1 & \text { for } j=d \\
0 & \text { for } j \neq d \text { and } j \neq s \\
-1 & \text { for } j=s
\end{array}\right.
\end{gathered}
$$

The set $\left\{f_{i j}^{s d}\right\}$ univocally defines the corresponding routing strategy in the network/graph.

Unfortunately, MCF problems are, in general, hard to solve; an upper bound to $\lambda$ can be obtained in terms of graph cuts:

Proposition 2: Traffic $\lambda \boldsymbol{\Lambda}$ is sustainable only if, for any partition $(\mathcal{S}, \mathcal{D})$ of the nodes, it results:

$$
\lambda \sum_{s \in \mathcal{S}} \sum_{d \in \mathcal{D}} \lambda_{s d} \leq \sum_{i \in \mathcal{S}} \sum_{j \in \mathcal{D}} \mu_{i j}^{n}
$$

It has been proven in [24] that, in undirected graphs, traffic is guaranteed to be sustainable if the ratio between the minimum value of the r.h.s. and the maximum value of the 1.h.s. in (7) is $\Omega(\log k)$, being $k$ the number of flows.

\footnotetext{
${ }^{6}$ Indeed, notice that i) transmission between nodes residing in the same cluster are not effective to make information advance along its path $s \rightarrow d$ in graph $G(V, E, \mu)$; ii) if one selects $R_{T}=\Theta(d)$ the system performance gets severely degraded for effect of interference, resulting in the same per-node capacity as that of a static network, i.e., $\Theta\left(n^{\nu / 2-1}\right)$
} 